\title{
A CLASS OF DIFFERENCE KERNELS
}

\section{MARVIN SHINBROT}

1. Introduction. Tricomi's statement $[1$, p. 143] notwithstanding, very little is known about integral equations of the first kind. So little is known, in fact, that to say anything at all about solutions requires that the kernels be very special.

The type of kernel that leads to the most elegant results is the difference kernel $[2 ; 3 ; 4]$. If the range of validity of an integral equation with a difference kernel is either the whole real line or a semiinfinite interval, definitive results are known $[2 ; 3 ; 5 ; 6]$. On the other hand, when the range is only a finite interval, so that the problem becomes the solution of

$$
\phi(x)=\int_{-1}^{1} k(|x-y|) f(y) d y, \quad-1<x<1,
$$

general theorems on the existence of solutions have only recently been derived [4].

In order to study the pathology of (1.1), it is helpful to have on hand a stock of examples that can be solved explicitly. It is the purpose of this note to derive an inversion formula for (1.1) for a certain class of kernels. The application of this formula to show that some of the results of [4] are best possible will be reserved for a later paper.

Let $k \in L$, and let $^{1} J$ denote the reciprocal of the Fourier transform of $k(|x|)$. To invert (1.1), it will be assumed that

(i) $J(w)$ is an entire function of exponential type $t<2$;

(ii) $J(w)$ has no real zeroes;

(iii)

$$
\int_{-\infty}^{\infty} \frac{\log |J(w)|}{1+w^{2}} d w<\infty
$$

(iv) The quantities

$$
\begin{aligned}
& \lambda \equiv \limsup _{w \rightarrow \infty ; w \text { real }} \frac{\log |J(w)|}{\log w}, \\
& \mu \equiv \liminf _{w \rightarrow \infty ; w \text { real }} \frac{\log |J(w)|}{\log w}
\end{aligned}
$$

Received by the editors January 23, 1961 and, in revised form, April 7, 1961.

1 The range of the argument of $k$ in $(1.1)$ is the interval $(-2,2)$. It is tacitly assumed here that $k$ has some extension over the rest of the line such that $J$ exists and satisfies (i)-(iv). 
are both finite.

In view of (ii) and the fact that $k(|x|)$ is even, no loss of generality will be involved if we assume

$$
J(w)>0
$$

on the real axis. We mention here explicitly that (1.2) will be assumed in all that follows.

2. Notation. Functions of the independent variable $x$ (or $y$ ) will be denoted by lower case letters. Given a function of $x$, denoted by some lower case letter, the same letter in upper case will be used to denote its Fourier transform.

Given a function $f(x)$, by $f_{a}^{b}(x)$ we shall mean the function

$$
f_{a}^{b}(x)= \begin{cases}f(x), & a<x<b, \\ 0, & \text { otherwise. }\end{cases}
$$

The Fourier transform of $f_{a}^{b}$ will be denoted by $F_{a}^{b}$. Notice that $F_{a}^{b}$ is not zero outside $(a, b)$; only its inverse transform has this property.

The utility of the notation introduced in the last paragraph results from the validity of the obvious

Lemma 2.1. Consider two transforms $F_{a}^{b}, G_{c}^{d}$. If the product

$$
H=F_{a}^{b} G_{c}^{d}
$$

is a transform, then

$$
H=H_{a+c}^{b+d}
$$

The result for the lower index, say, results from the fact that a transform $H=H_{\beta}^{\infty}$ if and only if it analytic and $O\left(e^{i \beta w}\right)$ in an upper half of the complex w-plane [2].

We shall frequently have occasion to utilize functions of $w$ that are not transforms. (The function $J$ of $\$ 1$ is an example.) If such a function has the appropriate analyticity properties and increases no faster than a power of $w$ as $\operatorname{Re} w$ approaches infinity, the notation of the second paragraph of this section will still be applied to it. Thus, with this convention, (i) and (iv) give

$$
J=J_{-t}^{t}
$$

The point is that if some $G_{a}^{b}$ approaches zero fast enough at infinity, the product $J_{-t}^{t} G_{a}^{b}$ will be a transform, even though $J_{-t}^{t}$ is not, and, because of the analyticity properties of the product, Lemma 2.1 is applicable to it, so that 


$$
J_{-t}^{t} G_{a}^{b}=\left(J_{-t}^{t} G_{a}^{b}\right)_{a-t}^{b+t}
$$

3. Factorization of $J$. It is (essentially) shown in [7] that if $J$ satisfies (i), (ii), and (iii), then there exist two entire functions, $L_{0}^{z}$ and $L_{-t}^{0}$, satisfying the conditions

$$
\begin{aligned}
L_{-t}^{0} L_{0}^{t} & =J_{-t}^{t}, \\
L_{-t}^{0}(w) & =\left[L_{0}^{t}(\bar{w})\right]^{-}
\end{aligned}
$$

(the bar denoting the complex conjugate), and

$$
L_{0}^{t}(w) \text { has no zeroes for } \operatorname{Im} w \geqq 0 \text {. }
$$

It follows from (3.1), (3.2) and (1.2) that

$$
\left|L_{0}^{t}\right|=\left|L_{-t}^{0}\right|=\left[J_{-t}^{t}\right]^{1 / 2}
$$

whenever $w$ is real. In view of (iv), then, we have that whenever $\epsilon>0$,

$$
\left|L_{0}^{t}\right|=\left|L_{-t}^{0}\right|=O\left(w^{(\lambda+\epsilon) / 2}\right)
$$

and

$$
\frac{1}{\left|L_{0}^{t}\right|}=\frac{1}{\left|L_{-t}^{0}\right|}=O\left(w^{-(\mu-\epsilon) / 2}\right)
$$

as $|w| \rightarrow \infty$ along the real axis.

According to (3.3), $1 / L_{0}^{t}$ is analytic and of exponential type zero in the upper half-plane Im $w \geqq 0$. Thus, by (3.6), the Phragmén-Lindelöf theorem can be applied to the function

$$
\frac{(1-i w)^{(\mu-\epsilon) / 2}}{L_{0}^{t}(w)}
$$

to show that for every $\epsilon>0$

(3.7) $\frac{1}{\left|L_{0}^{t}(w)\right|}=O\left(w^{-(\mu-\epsilon) / 2}\right)$ as $|w| \rightarrow \infty$ in the upper half-plane.

By (3.2), a similar result holds fof $L_{-t}^{0}$ in the lower half-plane.

4. Inversion of (1.1). We can now state our main theorem:

Let $J(w)$ satisfy (i)-(iv). Then, (1.1) has a unique solution whenever $\phi(x) \in C^{2}[-1,1]$ and $\lambda<2$. This solution is in $L^{p}$ for all $p<2 / \lambda$. Furthermore, its Fourier transform is given by the formula

$$
F_{-1}^{1}=J_{-t}^{t} \Phi_{-1}^{1}-L_{-t}^{0}\left(L_{0}^{t} \Phi_{-1}^{1}\right)_{1}^{\infty}=L_{0}^{t}\left(L_{-t}^{0} \Phi_{-1}^{1}\right)_{-\infty}^{-1}
$$


Finally, the condition $\lambda<2$ is best possible.

By the last sentence we mean that if $\lambda \geqq 2$ a solution may not exist. ${ }^{2}$ To prove it, it is only necessary to consider the example $J=1+w^{2}$, $\phi=1,-1 \leqq x \leqq 1$.

To prove the main part of the theorem, let $\Psi$ be any continuous function of $w$ satisfying the following conditions:

$$
\begin{aligned}
\Psi(w) & =O\left(\frac{1}{w^{2}}\right) \text { as }|w| \rightarrow \infty \text { along the real axis, } \\
\Psi_{-1}^{1} & =\Phi_{-1}^{1} .
\end{aligned}
$$

That such a function exists is easy to see, for let $\psi(x)$ be any twice continuously differentiable function with compact support such that

$$
\psi(x)=\phi(x), \quad-1 \leqq x \leqq 1 .
$$

Then, if $[-a, a]$ is any interval containing the support of $\psi$,

$$
\Psi(w)=\int_{-a}^{a} \psi(x) e^{i w x} d x .
$$

(4.3) follows from (4.4). Also, (4.2) follows from two by parts integrations of (4.5) and the Riemann-Lebesgue lemma.

On the real axis, the functions $L_{0}^{t} \Psi$ and $L_{-i}^{0} \Psi$ are continuous and $O\left(w^{-2+(\lambda+\epsilon) / 2}\right)$, by (3.5) and (4.2). Since $\lambda<2$, then, these functions are transforms [2], and the expressions $\left(L_{0}^{t} \Psi\right)_{1}^{\infty}$ and $\left(L_{-t}^{0} \Psi\right)_{-\infty}^{-1}$ have unique meanings.

Consider the function

$$
F=J_{-t}^{t} \Psi-L_{-t}^{0}\left(L_{0}^{t} \Psi\right)_{1}^{\infty}-L_{0}^{t}\left(L_{-t}^{0} \Psi\right)_{-\infty}^{-1} .
$$

We shall show that $F$ is a transform. First of all, $F$ is entire, for, by (4.5), $\Psi=\Psi_{-a}^{a}$, so that

$$
F=J_{-t}^{t} \Psi_{-a}^{a}-L_{-t}^{0}\left(L_{0}^{t} \Psi_{-a}^{a}\right)_{1}^{t+a}-L_{0}^{t}\left(L_{-t}^{0} \Psi_{-a}^{a}\right)_{-t-a}^{-1},
$$

by Lemma 2.1. Thus, $F$ is a sum of products of entire functions and is itself entire. According to the $L^{q}$ version of the Paley-Wiener theorem [7], then, to prove that $F$ is a transform it will be sufficient to prove that for every $\epsilon>0$,

$$
F(w)=O\left(w^{-1+\lambda / 2+\epsilon}\right)
$$

on the real axis.

${ }^{2}$ However, if distributions (in the sense of L. Schwartz) are allowed as solutions, one will always exist. See [4]. 
The first term on the right of (4.6) satisfies (4.7) trivially because of (4.2), (iv), and the assumption that $\lambda<2$. Because of (3.5), then, (4.7) will follow if it can be shown that $\left(L_{0}^{t} \Psi\right)_{1}^{\infty}$ and $\left(L_{-t}^{0} \Psi\right)_{-\infty}^{-1}$ are $O\left(w^{-1+\varepsilon}\right)$. Because of $(3.2)$, however, it will be enough to prove the result for the function $\left(L_{0}^{t} \Psi\right)_{1}^{\infty}$. Thus, we shall show that

$$
\left(L_{0}^{t} \Psi\right)_{1}^{\infty}=O\left(w^{-1+\epsilon}\right) \text { as }|w| \rightarrow \infty \text { along the real axis. }
$$

$L_{0}^{t} \Psi$ itself is $O\left(w^{-2+(\lambda+\epsilon) / 2}\right)$, as we have already seen. Since $\lambda<2$, then, $L_{0}^{t} \Psi$ is surely $O\left(w^{-1+\varepsilon}\right)$ for every $\epsilon>0$. Since $L_{0}^{t} \Psi$ is continuous, therefore, we may write

$$
\left|L_{0}^{t} \Psi\right| \leqq \frac{c}{1+|w|^{1-c}}
$$

on the real axis, where $c$ is a constant.

Now, $\left(L_{0}^{t} \Psi\right)_{1}^{\infty}=\left(L_{0}^{t} \Psi\right)_{1}^{t+a}$, so that

$$
\left(L_{0}^{t} \Psi\right)_{1}^{\infty}=\frac{1}{2 \pi i} \int_{-\infty}^{\infty} L_{0}^{t}(z) \Psi(z) \cdot \frac{e^{i(t+a)(w-z)}-e^{i(w-z)}}{w-z} d z,
$$

and, by (4.9),

$$
\left|\left(L_{0}^{t} \Psi\right)_{1}^{\infty}\right| \leqq \frac{c}{\pi 2^{1 / 2}} \int_{-\infty}^{\infty}\left[\frac{[1-\cos (t+a-1) w z]^{1 / 2}}{|z|}\right] \frac{d z}{1+|w(z+1)|^{1-c}} .
$$

If the term in brackets is replaced by $2^{1 / 2} /|z|$ when $|z|>1$ and by $2^{1 / 2}$ when $|z|<1$, a simple calculation can be used to derive (4.8). Then, (4.7) and the fact that (4.6) is a transform follow.

We note also that (4.7) implies $F \in L^{a}$ whenever $q>2 /(2-\lambda)$. Consequently, $f$, the inverse transform of $F$, is in $L^{p}$ whenever $p<2 / \lambda$, by the Paley-Wiener theorem.

Also, $F=F_{-1}^{1}$, for, writing

$$
\left(L_{0}^{t} \Psi\right)_{1}^{\infty}=L_{0}^{t} \Psi-\left(L_{0}^{t} \Psi\right)_{-\infty}^{1}
$$

in (4.6), we see that

$$
F=L_{-t}^{0}\left(L_{0}^{t} \Psi\right)_{-\infty}^{1}-L_{0}^{t}\left(L_{-t}^{0} \Psi\right)_{-\infty}^{-1} .
$$

Since $t<2$, Lemma 2.1 gives the result that $F_{1}^{\infty}=0$. Similarly, writing

$$
\left(L_{-t}^{0} \Psi\right)_{-\infty}^{-1}=L_{-t}^{0} \Psi-\left(L_{-t}^{0} \Psi\right)_{-1}^{\infty}
$$

we find that $F_{-\infty}^{-1}=0$ and $F=F_{-1}^{1}$.

Consider 


$$
\frac{F_{-1}^{1}}{J_{-t}^{t}}=\Psi-\frac{1}{L_{0}^{t}}\left(L_{0}^{t} \Psi\right)_{1}^{\infty}-\frac{1}{L_{-t}^{0}}\left(L_{-t}^{0} \Psi\right)_{-\infty}^{-1} .
$$

$\left(L_{0}^{t} \Psi\right)_{1}^{\infty}$ is entire, as we have seen. By (4.8) and the PhragménLindelof theorem, it is easy to see that

$$
\left(L_{0}^{t} \Psi\right)_{1}^{\infty}=O\left(w^{-1+\epsilon} e^{i w}\right)
$$

as $|w| \rightarrow \infty$ in the upper half-plane. Also, $1 / L_{0}^{\ell}$ is analytic in the upper half-plane, by (3.3), and satisfies (3.7). Thus, the second term on the right of (4.10) is analytic and $O\left(w^{-1-\mu / 2+e} e^{i v}\right)$ in the upper half-plane. Let $\delta$ be positive and consider

$$
\left[\frac{1}{L_{0}^{t}}\left(L_{0}^{t} \Psi\right)_{1}^{\infty}\right]_{-\infty}^{1-\delta}=\frac{1}{2 \pi i} \int_{-\infty}^{\infty} \frac{e^{i(1-\delta)(w-z)}}{w-z} \cdot \frac{1}{L_{0}^{t}}\left(L_{0}^{t} \Psi\right)_{1}^{\infty} d z
$$

whenever Im $w<0$. The last integral is zero, a fact that can be seen by closing the contour in the upper half of the complex z-plane and using the order relation just derived.

In a similar way, it can be shown that

$$
\left[\frac{1}{L_{-t}^{0}}\left(L_{-t}^{0} \Psi\right)_{-\infty}^{-1}\right]_{-1+8}^{\infty}=0
$$

Therefore, (4.10) gives

$$
\begin{aligned}
\left(\frac{F_{-1}^{1}}{J_{-t}^{t}}\right)_{-1+\delta}^{1-\delta} & =\Psi_{-1+\delta}^{1-\delta} \\
& =\Phi_{-1+\delta}^{1-\delta}
\end{aligned}
$$

by (4.3). Since $k$, the inverse transform of $1 / J$, is in $L$ and $F_{-1}^{1} \in L^{q}$, it follows [2] that $f(x)$ satisfies (1.1) whenever $|x|<1-\delta$. Since $\delta$ was arbitrary, however, we see that $f$ is really a solution of (1.1).

We shall now show that (4.1) and (4.6) define the same function $F$. To do so, we note that $\Psi_{1}^{\infty}$ is $O(1 / w)$ on the real axis, since we have

$$
\begin{aligned}
\Psi_{1}^{\infty} & =\int_{1}^{a} e^{i w x} \psi(x) d x \\
& =-\psi(1) \frac{e^{i w}}{i w}-\frac{1}{i w} \int_{1}^{a} e^{i w x} \psi^{\prime}(x) d x .
\end{aligned}
$$

Thus, $L_{0}^{\imath} \Psi_{1}^{\infty}$ and $L_{-t}^{0} \Psi_{1}^{\infty}$ are entire and belong to $L^{q}$ on the real axis. They are, therefore, transforms, so that we may write

$$
\left(L_{0}^{t} \Psi_{1}^{\infty}\right)_{1}^{\infty}=L_{0}^{t} \Psi_{1}^{\infty},
$$


and

$$
\left(L_{-t}^{0} \Psi_{1}^{\infty}\right)_{-\infty}^{-1}=0 \text {, }
$$

by Lemma 2.1 , since $t<2$. In the same way, we find that

$$
\begin{aligned}
\left(L_{0}^{t} \Psi_{-\infty}^{-1}\right)_{1}^{\infty} & =0, \\
\left(L_{-t}^{0} \Psi_{-\infty}^{-1}\right)_{-\infty}^{-1} & =L_{-t}^{0} \Psi_{-\infty}^{-1} .
\end{aligned}
$$

Thus, (4.6) and (4.3) give (4.1).

It remains only to show that the solution we have found is unique. Let $f \in L$ be any solution of (1.1) with $\phi(x)=0,|x|<1$. If $\phi(x)$ is defined by the right side of (1.1) for $|x| \geqq 1$, we find that

$$
F_{-1}^{1}=J_{-t}^{t}\left(\Phi_{-\infty}^{-1}+\Phi_{1}^{\infty}\right) \text {, }
$$

so that

$$
\begin{aligned}
J_{-t}^{t}\left(\Phi_{-\infty}^{-1}+\Phi_{1}^{\infty}\right) & =\left[J_{-t}^{t}\left(\Phi_{-\infty}^{-1}+\Phi_{1}^{\infty}\right)\right]_{-1}^{1} \\
& =0,
\end{aligned}
$$

again by Lemma 2.1. It follows that $F_{-1}^{1}=0$.

5. Some examples. Before closing, it will probably be well to demonstrate that our theorem is not completely vacuous. We shall do this here by displaying some examples.

The simplest functions that satisfy the hypotheses (i)-(iv) are, of course, the even polynomials without real zeroes. For these functions, however, $\lambda$ is at least two, so that the main theorem does not apply. (On the other hand, as noted in footnote 1, distributional solutions of (1.1) exist even when $\lambda \geqq 2$. Furthermore, the formula (4.1), when interpreted carefully, continues to give the solution in these cases.)

A function that satisfies (i)-(iv) with $\lambda<2$ is

$$
J(w)=\left[(i-w)^{1 / 2} \sin (i-w)^{1 / 2}\right]\left[(i+w)^{1 / 2} \sin (i+w)^{1 / 2}\right],
$$

where by $z^{1 / 2} \sin z^{1 / 2}$ we mean the entire function defined by the series

$$
\sum \frac{(-z)^{n}}{(2 n+1) !}
$$

(5.1) obviously satisfies (i) and (ii). Also, $\lambda=\mu=1$, so that (iv)-and, as a consequence, (iii)-is satisfied.

It is not really necessary for the validity of our theorem, but we have assumed that the kernel $k$ is in $L$. (We only need that $k$ have a Fourier transform in some sense and that the convolution theorem 
hold.) For completeness, therefore, it will now be shown that the kernel corresponding to (5.1) belongs to $L$. To show this, we note that $1 / J$ is analytic for $|\operatorname{Im} w|<1$. Thus, by a slight generalization of an argument to be found in [2], $k=O\left(e^{-|x|}\right)$ as $|x| \rightarrow \infty$. Also, $1 / J \in L^{2}$. Thus [2], $k \in L^{2}$, so that $k \in L$ in any finite interval. The result now follows.

A final example of a function satisfying our hypotheses is

$$
J(w)=\left(1+w^{2}\right) \int_{0}^{t} \frac{\cos w x}{x^{1 / 2}} d x .
$$

For this function, (i) is immediate. (ii), (iii), and (iv) (with $\lambda=\mu=3 / 2$ ) follow from known properties [8] of the Fresnel integral, to which (5.2) is closely related.

If $J$ is given by (5.2), it is rather hard to see whether or not the corresponding kernel is in $L$. On the other hand, $1 / J \in L^{p}$ (all $p \geqq 1$ ) since $\mu=3 / 2$. Furthermore, the function $F_{-1}^{1}$ of $(4.1)$ is in $L^{q}$ whenever $q>2 /(2-\lambda)=4$, by the same proof as before. It follows from these two facts [2] that the convolution theorem applies to the product $(1 / J) F_{-1}^{1}$, so that the inverse transform of $F_{-1}^{1}$ satisfies (1.1).

\section{REFERENCES}

1. F. G. Tricomi, Integral equations, Interscience, New York, 1957.

2. E. C. Titchmarsh, Introduction to the theory of Fourier integrals, 2nd ed., Oxford, 1948.

3. I. I. Hirschman and D. V. Widder, The convolution transform, Princeton Univ. Press, Princeton, N. J., 1955.

4. Marvin Shinbrot, Difference kernels, Stanford Univ. Appl. Math. and Stat. Labs., Tech. Rep. no. 92, 1960.

5. Norbert Wiener, Interpolation, extrapolation, and smoothing of stationary time series, Wiley, New York, 1947.

6. B. Noble, The Wiener-Hopf technique, Pergamon, New York, 1958.

7. Ralph P. Boas, Jr., Entire functions, Academic Press, New York, 1954.

8. A. Erdélyi et al., Higher transcendental functions, Vol. I, McGraw-Hill, New York, 1953.

STANFORD UNIVERSITY 